\title{
Simulation of defect zones in scribed silicon wafers
}

\author{
Alexey I Ogorodnikov, Olga M Ogorodnikova and Igor N Tikhonov \\ Ural State Technical University, Mira Street, 19, Ekaterinburg 620002, Russia \\ E-mail: o.m.ogorodnikova@bk.ru
}

\begin{abstract}
The paper presents the results of computer simulation of silicon wafers under scribe loading conditions. Finite Element (FE) analysis was applied to estimate a value of stresses and spread of defect zone around scratch line. It was revealed that due to impact of diamond tip, a complex stress-strain state is produced in the wafer, which is related to the appearance of defect zones in silicon. The approved methods of cutting simulation could be employed for various types of brittle materials to predict defects and damage of crystal during separation processing.
\end{abstract}

\section{Introduction}

Silicon remains the most common semiconductor and construction material used in different devices on a base of Micro-Electro-Mechanical Systems (MEMS). It takes place because silicon is a suitable platform on which electronic, mechanical, thermal, optical and fluid-flow functions can be integrated [1]. Recently, the computer-aided design grounded by simulation of structures and technologies seems to become the obligatory start point of manufacturing the devices [2]. There is advanced FE software to simulate all the aspects of MEMS through multiphysics modelling [3].

Various techniques related to MEMS have been developed to fabricate units on silicon-based materials. Dicing of silicon wafers is an important preparation technology [4]. Wafer is a thin circular slice cut from the ingot. Wafer dicing is the process of division a wafer into separate dies. Historically, the first method of division was to scribe a line with a diamond and then to break the wafer along the scribed line. Diamond scribe, laser scribe, stealth dicing, and blade half-cutting are in use today. Owing to technical innovation and demand for thinner and higher grade dies, the dicing process has been changing. In any case, dicing technique affects on the mechanical behavior of silicon wafers [5], the phenomenon becomes apparent when silicon intends for projection smaller and finer structures [6].

Die separation process could be accomplished by diamond scribing and mechanical breaking. Thereupon dicing of the wafer is performed in two stages. The surface is preliminarily scratched with a sharp diamond tip, which creates stress in a wafer and induces structural defects. The purpose of the scribe is to initiate the crack for the break along the desired cleavage plane. Then, by applying pressure loading on the backside of the wafer, the crack propagation is initiated, starting from the defect area till the total separation. Scribe \& break uses crystal cleavage planes to advantage so that less physical cutting is needed and, hopefully, less damage occurs to the wafer [7]. The features of scribe \& break die separation are especially beneficial with thin silicon wafers; delicate III-V materials; and hard materials, including sapphire, glass and ceramics.

Computer prediction of defects and damage in a crystal over technological processing could ensure high quality of devices. However, the mechanism leading to perfect surfaces of definitive die is not completely understood. The purpose of this study was to simulate scribing of silicon wafer and to analyse its behavior under the loading condition. 


\section{Methodology}

Computer simulation of silicon plates under scribe loading conditions was performed through FE modelling to estimate value of stresses and spread of defect zone around scratch line. APDL programming in FE software package ANSYS has been used to solve this simulation task. For the first estimation, the two-dimensional FE model was build reasoning from axisymmetry of a circle wafer geometry. The model consists of silicon plate glued to flexible film and diamond indenter with a tip radius about $1 \mu \mathrm{m}$. The model assumes a steady-state statical condition and is meshed using $2 \mathrm{D}$ eightnode solid element, as shown in Fig. 1. Loading and constraints include fixed support along lower film surface and forced displacement of indenter down. For a given displacement, the total load was extracted from the output as the total reaction force acting on the rigid indenter. A fine mesh as well as contact elements were equipped at the region around diamond tip in order to increase the accuracy of stress computation and decrease the square of singularity effect. On the contrary, a coarser mesh was used at the areas away from the contact region in order to reduce the total number of elements in the model.

Some full-scale experiments were carried out. Scribe lines on the surface of wafers were created using special equipment with ability to load the tip by a force up to $1 \mathrm{~N}$. Profiles of 3D surface's geometry were pictured using profilometer and simultaneously $2 \mathrm{D}$ traces along or crosswise the scratch were measured. Scratch-induced mechanical damage on silicon surfaces was observed by means of optical microscopy.

\section{Results and discussion}

It is known, that the single crystal silicon has a diamond-like lattice structure with a covalent bond, hence leading to its nature of being hard and brittle. It is possible to scribe silicon wafer by the diamond tip due to higher hardness of a diamond crystal. Scribing with a diamond locally generates the concentrated mechanical stress in the wafer owing to the sharpness of the diamond tip. Fig. 2 shows distribution of stresses in the immediate region under the tip computed on the assumption of penetration of diamond into the silicon surface.

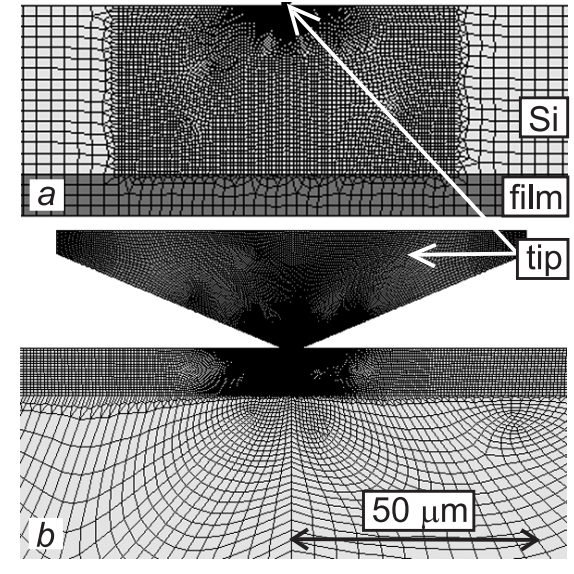

Figure 1. FE mesh throughout the height $(a)$ and its magnified image in the region of the tip $(b)$
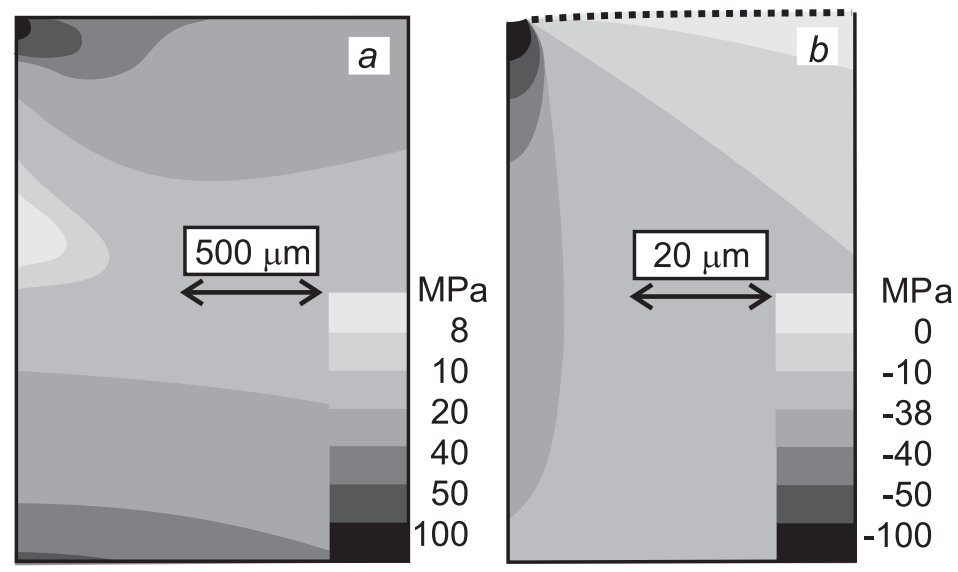

Figure 2. Distribution of stresses - stress intensity $\sigma_{I}$ throughout the height of wafer $(a)$ and major principal stress $\sigma_{1}$ in the immediate region under the tip $(b)$

Indentation-induced tensile stresses assessed through a detailed numerical investigation could clarify the crack initiation and propagation, therefore stress examination serves as the first step prior to a full fracture mechanics analysis [8]. As a rough and first approximation of the process the spherical Hertzian contact theory could be applied to describe the behavior of diamond indenter that is loading isotropic surface inside the elastic interval. On a basis of such analysis it could be concluded that during contact of circular indenter, a complex stress state that is related to the material deformation is produced in wafer. 
The indentation stress field, although dominated by the shear and compression components, nevertheless contains an area of tension, which creates the conditions for cracking. Parameter of stress intensity represents the maximum shear stresses. The stress intensity $\sigma_{I}$ is the largest of the absolute values of principal stresses differences $\sigma_{1}-\sigma_{2}, \sigma_{2}-\sigma_{3}$, or $\sigma_{3}-\sigma_{1}$. That is: $\sigma_{I}=\operatorname{MAX}\left(\left|\sigma_{1}-\sigma_{2}\right| ; \mid \sigma_{2}-\right.$ $\left.\sigma_{3}|;| \sigma_{3}-\sigma_{1} \mid\right)$. Scribing induces singular deformation of the wafer. It can be seen that the compression zone with high negative values of major principal stress $\sigma_{1}$ occurs in the wafer just under the diamond tip. On a contrary, the tensile stress area is located at the surface and has a form of a very narrow horizontal layer about $1 \mu \mathrm{m}$ wide, this area is pictured on Fig. 2 by dotted line.

There is a necessary condition for cracking of silicon as a consequence of loading by sharp indenter, because silicon exhibits high strain hardening, that means, a relatively high hardness to Young's modulus ratio. So the wafer breaks along scribe line after applying force afterwards. The better initial condition for brittle fracture (also called cleavage) following the scribing is the existence of deep and narrow scratch locally concentrating the stress. But, the depth of the cut is not as important as the sharpness of the cut. Measured 3D profile of wafer surface and 2D traces crosswise the scratch are shown in Fig. 3. The diamond scribe creates a V-profile in the wafer surface, and the width of the scribe on the surface is about $30 \mu \mathrm{m}$. Measured depth of the cut is not equal along the scratch and is found to be in a range about 3 to $5 \mu \mathrm{m}$ deep.
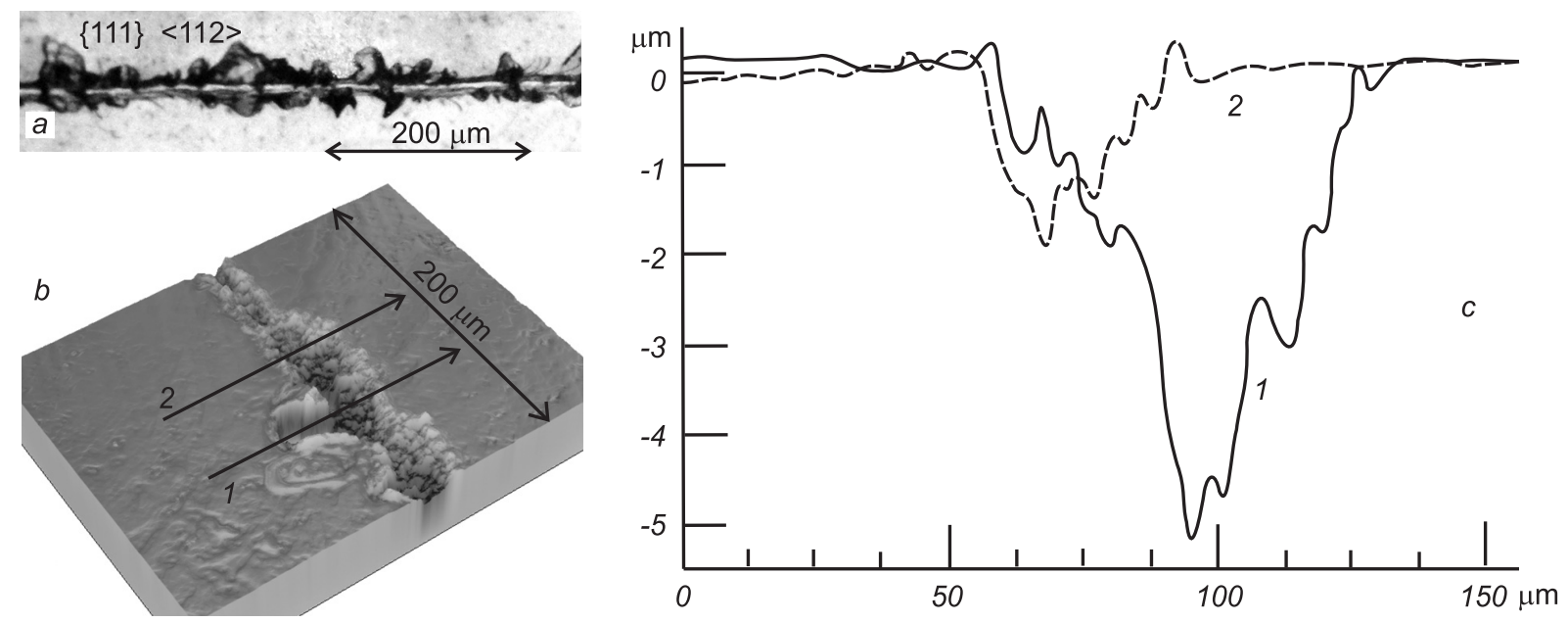

Figure 3. Optical microscope image of the scratch produced by Berkovich indenter on a surface of $\{111\}$-oriented silicon wafer in $\langle 112\rangle$ direction $(a)$, 3D profile of scribed surface $(b)$, and 2D traces across the scratch $(c)$

The scratch shown on Fig. 3 was produced by the Berkovich indenter on a surface of $\{111\}$-oriented silicon wafer in $\langle 112\rangle$ direction. The loading force was $1 \mathrm{~N}$, and axis of the cutting tool was oriented normally to the cut surface. The Berkovich indenter tip is a three-sided pyramid that can be ground to a point and thus maintains a self-similar geometry to very small scales. The Berkovich indenter tip has a large included angle of $142.3^{\circ}$ which minimizes the influence of friction. The geometry of Berkovich indenter is similar to the shape of a lathe cutting tool, so its moving and impact could be described by analogy with the cutting theory for brittle materials. In that way it's possible to describe the mechanism of ductile chip formation shown on Fig. 3. It's known, the ductile chip formation is a result of large compressive and shear stresses in the chip formation zone, which shields the growth of pre-existing flaws [9]. Large compressive stress can be generated in the chip due to small value of undeformed chip thickness being smaller than the radius of the tool cutting edge.

Silicon single-crystalline wafers mostly have $\{100\}$ or $\{111\}$ orientation of top surface faces. The single-crystal silicon MEMS devices fabricated using \{111\}-wafers are least sensitive to device 
orientation with respect to crystallographic orientations, therefore the $\{111\}$-wafers are very convenient for many applications. In this work the $\{111\}$-oriented silicon wafers were examined.

We could embody the scribing process like constrained skew-angular cutting of single crystal by means of three-edged cutting tool with sharp cutter point. Following the diamond tip movement the defects could arise around scribe line, i.e. dislocations, microcracks and chips. The defects appear due to complicated mode of deformation which is not a plain one. The quantity and dimensions of defects is determined by mechanism of scratching, which depends on shape of diamond tip, the scratching direction, the moving velocity of the diamond tip and applied loads.

Appearance of the scratch is not a rigorous criterion of residual stress value close to scribe line. Deformative area along the scratch looks as ragged band, but the stress breadth here is rather low. Surface chips (Fig. 3) implement destroying stress and generate low stressed state of crystal integrally. Asymmetrical processing could bring good quality of the surface.

Cracks on the wafer mainly propagate along the development of the easiest slip direction family $\langle 110\rangle$, and the lateral cracks are situated along the easiest cleavage plane family $\{111\}$. Silicon scribe and break is more difficult to break at a $90^{\circ}$ angle to the scribe due to the surface plane $\{111\}$ being favored for electronic applications. The natural set of $\{111\}$ cleavage planes are at $54.7^{\circ}$ to each other.

\section{Conclusion}

Computer simulation of plate samples under specific loading conditions was performed through FE modelling to estimate value of stresses and depth of defect zone under scratch line. On a base of such analysis it was concluded that during contact of indenter, a complex stress state that is related to the silicon deformation is produced in wafer. The application of numerical techniques, such as the FE analysis to cutting problems, enhances understanding and promotes further development of existing separation techniques. The results and approved methods could be employed to various types of brittle materials.

It is of interest to attempt to extend the analysis to the case where the Berkovich indenter is moved across the surface of 3D FE model under different condition.

\section{References}

[1] Maluf N and Williams K 2004 An Introduction to Microelectromechanical Systems Engineering (Boston: Artech House)

[2] Ogorodnikova O M and Panin O A 2009 Nano and Microsystem Technique 512

[3] Ladani L J 2010 Microelectronic Engineering 871852

[4] Sullivan S 2010 Handbook of Silicon Based MEMS Materials and Technologies (Oxford: Elsevier) chapter 41 pp 601-606

[5] Schoenfelder S, Ebert M, Landesberger C, Bock K and Bagdahn J 2007 Microelectronics Reliability 47168

[6] Wang T H, Fang T H and Lin Y C 2007 Materials Science and Engineering A 447244

[7] Cooke M 2006 III-Vs Review 1920

[8] Leonardi A, Furgiuele F, Woodb R J K and Syngellakis S 2010 Engineering Fracture Mechanics 77264

[9] Liu K, Li X P and Liang S Y 2007 Int J Adv Manuf Technol 33875 